\title{
Cost-effectiveness of mediastinal lymph node staging in non-small cell lung cancer
}

\author{
Katarzyna Czarnecka-Kujawa, MD, FRCPC, MPH, ${ }^{a}$ Ursula Rochau, MD, MSc, ${ }^{b}$ \\ Uwe Siebert, MD, MSc, MPH, ${ }^{\mathrm{b}, \mathrm{c}, \mathrm{d}, \mathrm{e}}$ Eshetu Atenafu, MSc, PStat, ${ }^{\mathrm{f}}$ Gail Darling, MD, \\ Thomas Kenneth Waddell, MD, MSc, PhD, ${ }^{a}$ Andrew Pierre, MD, MSc, ${ }^{a}$ Marc De Perrot, MD, MSc, ${ }^{a}$ \\ Marcelo Cypel, MD, MSc, ${ }^{a}$ Shaf Keshavjee, MD, MSc, ${ }^{a}$ and Kazuhiro Yasufuku, MD, PhD ${ }^{\mathrm{a}}$
}

\section{ABSTRACT}

Objective: To assess the cost-effectiveness of various modes of mediastinal staging in non-small cell lung cancer (NSCLC) in a single-payer health care system.

Methods: We performed a decision analysis to compare the health outcomes and costs of 4 mediastinal staging strategies: no invasive staging, endobronchial ultrasound-guided transbronchial needle aspiration (EBUS-TBNA), mediastinoscopy, and EBUS-TBNA followed by mediastinoscopy if EBUS-TBNA is negative. We determined incremental cost effectiveness ratios (ICER) for all strategies and performed comprehensive deterministic sensitivity analyses using a willingness to pay threshold of $\$ 80,000 /$ quality adjusted life year (QALY).

Results: Under the base-case scenario, the no invasive mediastinal staging strategy was least effective (QALY, 5.80) and least expensive $(\$ 11,863)$, followed by mediastinoscopy, EBUS-TBNA, and EBUS-TBNA followed by mediastinoscopy with 5.86, 5.87, and 5.88 QALYs, respectively. The ICER was $\sim \$ 26,000 /$ QALY for EBUS-TBNA staging and $\sim \$ 1,400,000 /$ QALY for EBUS-TBNA followed by mediastinoscopy. The mediastinoscopy strategy was dominated. Once pN2 exceeds $2.5 \%$, EBUS-TBNA staging is cost-effective ( $\sim 80,000 / \mathrm{QALY})$. Once the pN2 reaches $57 \%$, EBUS-TBNA followed by mediastinoscopy is costeffective (ICER $\sim \$ 79,000 /$ QALY). Once EBUS-TBNA sensitivity exceeds $25 \%$, EBUS-TBNA staging is cost-effective (ICER $\sim \$ 79,000 /$ QALY). Once pN2 exceeds $25 \%$, confirmatory mediastinoscopy should be added, in cases of EBUS-TBNA sensitivity $\leq 60 \%$.

Conclusions: Invasive mediastinal staging in NSCLC is unlikely to be costeffective in clinical $\mathrm{N} 0$ patients if $\mathrm{pN} 2<2.5 \%$. In patients with probability of mediastinal metastasis between $2.5 \%$ and $57 \%$ EBUS-TBNA is cost-effective as the only staging modality. Confirmatory mediastinoscopy should be considered in high-risk patients (pN2 $>57 \%$ ) in case of negative EBUS-TBNA. (J Thorac Cardiovasc Surg 2017;153:1567-78)

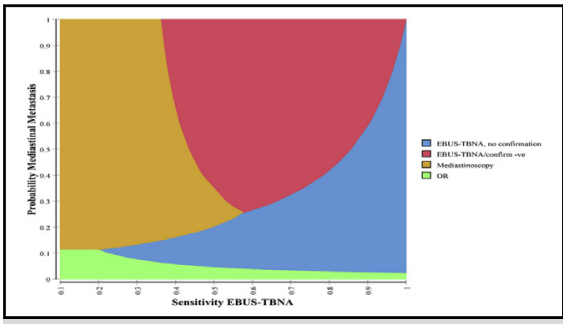

A 2-way sensitivity analysis on prevalence of mediastinal lymph node metastasis and endobronchial ultrasound transbronchial needle aspiration sensitivity.

\section{Central Message}

In the EBUS era it is cost-effective to invasively stage patients with early clinical NSCLC. The benefit of cervical mediastinoscopy following a negative EBUS becomes apparent in patients with more advanced disease.

\section{Perspective}

The choice of mediastinal lymph node staging strategy in patients with non-small cell lung cancer should be guided by the patient population characteristics (ie, clinical stage of the disease based on noninvasive staging), the local invasive staging test performance characteristics (ie, endobronchial ultrasound transbronchial needle aspiration or cervical mediastinoscopy), and the regional health economic data (ie, willingness to pay threshold).

See Editorial Commentary page 1579.

See Editorial page 1565 .

\footnotetext{
From the a Division of Thoracic Surgery, University of Toronto, Toronto General Hospital, University Health Network; ${ }^{b}$ Institute of Public Health, Medical Decision Making, and Health Technology Assessment, Department of Public Health, Health Services Research, and Health Technology Assessment, University of Health Sciences, Medical Informatics, and Technology, Tirol; ${ }^{~}$ Center for Personalized Cancer Medicine, Innsbruck, Austria; ${ }^{\mathrm{d}}$ Department of Health Policy and Management, Harvard T.H. Chan School of Public Health and Institute for Technology Assessment and Department of Radiology, Massachusetts General Hospital, Harvard Medical School; ${ }^{\mathrm{e}}$ Center for Health Decision Science, Boston, Mass; and ${ }^{\mathrm{f}}$ Biostatistics Department, Princess Margaret Cancer Center, University Health Network, Toronto, Ontario, Canada.
}

Read at the 96th Annual Meeting of The American Association for Thoracic Surgery, Baltimore, Maryland, May 14-18, 2016.

Received for publication June 20, 2016; revisions received Dec 7, 2016; accepted for publication Dec 17, 2016; available ahead of print March 7, 2017

Address for reprints: Katarzyna Czarnecka-Kujawa, MD, FRCPC, MPH, Division of Thoracic Surgery, Division of Respirology, University of Toronto, Toronto General Hospital, University Health Network, 585 University Ave, 9N 941, Toronto, Ontario, Canada (E-mail: kasia.czarnecka@uhn.ca). $0022-5223 / \$ 36.00$

Copyright (C) 2017 Published by Elsevier Inc. on behalf of The American Association for Thoracic Surgery

http://dx.doi.org/10.1016/j.jtcvs.2016.12.048 


\begin{tabular}{|c|c|}
\hline \multicolumn{2}{|c|}{ Abbreviations and Acronyms } \\
\hline CAD & $=$ Canadian dollar \\
\hline CRMM & $=$ cancer risk management model \\
\hline \multicolumn{2}{|c|}{$\begin{aligned} \text { EBUS-TBNA }= & \text { endobronchial ultrasound } \\
& \text { transbronchial needle aspiration }\end{aligned}$} \\
\hline ICER & $=$ incremental cost effectiveness ratic \\
\hline LN & $=$ lymph node \\
\hline MLNM & $=$ mediastinal lymph node metastasis \\
\hline MLNS & $=$ mediastinal lymph node staging \\
\hline NSCLC & $=$ non - small cell lung cancer \\
\hline QALY & $=$ quality adjusted life years \\
\hline VATS & $\begin{aligned}= & \text { video-assisted thoracoscopic } \\
& \text { surgery }\end{aligned}$ \\
\hline WTP & $=$ willingness to pay \\
\hline
\end{tabular}

Scanning this QR code will take you to a supplemental video for the article. To view the AATS 2016 Webcast, see the URL next to the video thumbnail.

Mediastinal lymph node ( $\mathrm{LN}$ ) assessment is a crucial step in lung cancer staging because it determines prognosis and guides management. Invasive mediastinal $\mathrm{LN}$ staging (MLNS) is recommended for patients with clinical stage
II disease, but not for patients with clinical stage I disease. This recommendation follows a widespread incorporation of positron emission tomography scan into the routine assessment of patients with non-small cell lung cancer (NSCLC). ${ }^{1}$

Given emerging evidence on improved outcomes in patients with micrometastatic N2 disease managed with trimodality approach over definitive chemoradiation, ${ }^{2,3}$ invasive mediastinal LN staging in patients with clinical stage I disease could be of benefit.

Introduction of needle-based techniques-endobronchial ultrasound transbronchial needle aspiration (EBUSTBNA), and esophageal ultrasound fine needle aspiration, revolutionized approach to MLNS in lung cancer, with the needle techniques being now recommended as test of first choice for invasive staging. ${ }^{1}$

Our study aim was to examine cost-effectiveness of various approaches to mediastinal $\mathrm{LN}$ assessment in patients with clinical N0 disease and T1 peripheral tumors using the Canadian health care system perspective. Our secondary aim was to develop a decision-analytic model to predict the optimal staging approach using the costeffectiveness data and performance characteristics of the various assessment algorithms (Video 1).

\section{METHODS}

\section{Decision-Analytic Framework}

We developed a decision tree model synthesizing evidence on clinical and economic events with their probabilities to simulate a cohort of hypothetical patients with biopsy-proven or suspected NSCLC with clinical NO disease. $^{4}$

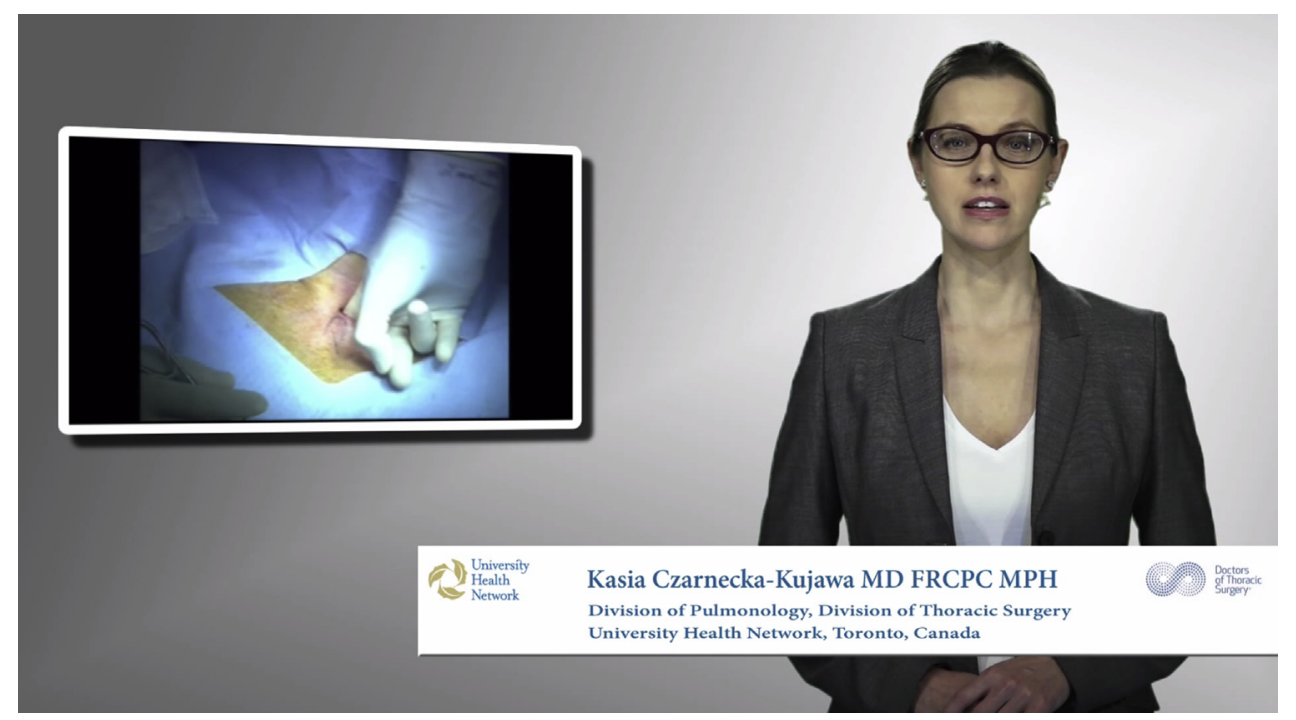

VIDEO 1. Dr Czarnecka-Kujawa explaining the importance of the work presented in this article to the thoracic community. Video available at: http://www. jtcvsonline.org/article/S0022-5223(17)30176-9/addons. 
TABLE 1. Prevalence of nodal metastasis

\begin{tabular}{lcc}
\hline \multicolumn{1}{c}{ Nodal status } & Prevalence & Reference \\
\hline N0 disease & 0.83 & 9 \\
N1 disease & 0.08 & 9 \\
N2 & 0.09 & 9 \\
N2 after EBUS-TBNA & 0.04 & 10 \\
\hline
\end{tabular}

EBUS-TBNA, Endobronchial ultrasound transbronchial needle aspiration.

We compared health outcomes and cost of 4 mediastinal staging strategies: no invasive staging, EBUS-TBNA, mediastinoscopy, and EBUSTBNA followed by mediastinoscopy if EBUS-TBNA is negative (EBUS-TBNA-mediastinoscopy). The analysis accounted for costs incurred as a result of procedures and treatments performed based on results of the noninvasive and invasive staging. Given the potential benefit of trimodality therapy in patients with micrometastatic mediastinal disease, we included this approach in our model. Patients with missed nodal metastasis were managed surgically followed by adjuvant chemotherapy.

Evaluated outcomes include quality-adjusted life years (QALY) over a lifetime horizon, costs, and incremental cost effectiveness ratios (ICER) in Canadian dollars (CAD) per QALY gained. A third-party payer perspective was adopted. Because the analysis focused on outcomes resulting from a diagnostic approach of a short duration and life expectancies of patients with NSCLC are relatively short, no discounting was applied.

We followed international modeling guidelines for model development ${ }^{5,6}$ and validation ${ }^{7}$ and international consolidated health economic evaluation reporting standards. ${ }^{8}$

\section{Modeling Assumptions}

Our model is based on the following assumptions:

- All patients were good surgical candidates and would tolerate a lobectomy,

- Patients with N2 disease on preoperative staging received trimodality therapy (neoadjuvant chemotherapy and radiation, followed by surgical resection),

- All nodal metastases were microscopic,

- Invasive staging techniques were $100 \%$ specific,

- All surgical resections were performed using the video-assisted thoracoscopic surgery (VATS) and only lobectomy or bilobectomy were included,

- All resections were $\mathrm{R} 0$, and

- No consolidation chemotherapy was performed.

\section{Model Parameters}

Clinical parameters. Prevalence of pathologic nodal metastasis was derived from published literature. ${ }^{9}$ For the base-case analysis, we assumed a prevalence of mediastinal metastasis of $9 \%$. Prevalence of pN2 disease following EBUS-TBNA was obtained from our institutional data $^{10}$ (Table 1).

TABLE 2. Life expectancies based on pN status

\begin{tabular}{lcl}
\hline \multicolumn{1}{c}{ Patient status } & Survival (y) & Reference \\
\hline Resection with pN0 & 7 & 36,37 \\
Resection with pN1 & 4.5 & 38,39 \\
Resection with pN2 (neoadjuvant) & 3.3 & 32 \\
Resection pN2 (adjuvant) & 2.2 & 40 \\
\hline
\end{tabular}

TABLE 3. Intervention costs

\begin{tabular}{lcl}
\hline \multicolumn{1}{c}{ Procedure } & Average cost* & Reference \\
\hline EBUS in the endoscopy & 1468 & $\mathrm{UHN}$ \\
Mediastinoscopy & 6633 & $\mathrm{UHN}$ \\
Mediastinoscopy death & 9702 & ${ }^{31} / \mathrm{UHN}$ \\
VATS & $11,215 \dagger$ & $\mathrm{UHN}$ \\
VATS death & 14,284 & ${ }^{15} / \mathrm{UHN}$ \\
Thoracotomy & 15,467 & ${ }^{31} / \mathrm{UHN}$ \\
Thoracotomy death & 18,536 & ${ }^{31} / \mathrm{UHN}$ \\
EBUS-TBNA in the OR & 8319 & ${ }^{31} / \mathrm{UHN}$ \\
EBUS in the OR death & 11,388 & ${ }^{31} / \mathrm{UHN}$ \\
Chemotherapy (neoadjuvant) & 4200 & 15 \\
Chemotherapy (adjuvant) & 3539 & 15 \\
Radiation & 4429 & 15 \\
Chemotherapy/radiation death & 5537 & 15 \\
\hline
\end{tabular}

EBUS, Endobronchial ultrasound; UHN, University Health Network; VATS, videoassisted thoracic surgery; TBNA, transbronchial needle aspiration; $O R$, operating room. *All costs have been adjusted to 2015 Canadian dollars (CAD) using the consumer price index (Bank of Canada). IIncludes the cost related to VATS complications (excluding operative death).

Health outcomes. Health status-specific life expectancies conditional on surviving index procedures were based on previously published literature (Table 2).

Health-related quality of life was based on quality-of-life indices (utilities). We used life expectancies and utilities to calculate QALY. ${ }^{11}$

To assign utility values for various stage-specific lung cancer health states specific to a Canadian population at diagnosis and during the treatment, a standardized tool was created by Statistics Canada: the Classification and Measurement System of Functional Health. ${ }^{12,13}$

Health care costs. We included initial and downstream cost based on the costing index year 2015. All cost data were adjusted for inflation to CAD for the price level of May of 2015 using the consumer price index from the Bank of Canada. ${ }^{14}$

Staging and procedure costs were based on recorded hospital costs of patients managed in our lung cancer program at the Toronto General Hospital between January 1, 2005, and December 31, 2014. In this database, a total of 4983 surgical costed cases were identified, of which 572 were thoracic surgery cases. We excluded nonlung cancer cases and cases performed with other than curative intent. The final case count was 499. The search of the endoscopy database identified 1044 thoracic surgery costed cases. We excluded cases performed for other-than-lung-cancer staging or diagnosis purposes, bringing the final count to 750 cases.

Mediastinoscopy at Toronto General Hospital is performed as a day procedure, under general anesthesia. EBUS-TBNA mediastinal staging at our hospital is a day procedure performed in an endoscopy suite under conscious sedation. Given that some programs perform EBUS-TBNA in the operating room, we evaluated that strategy in the same model as the other 4 strategies, and after having removed the option of performing EBUS-TBNA in the endoscopy suite.

TABLE 4. Sensitivities of diagnostic test procedures

\begin{tabular}{lccc}
\hline \multicolumn{1}{c}{ Intervention } & Sensitivity & Range & Reference \\
\hline EBUS-TBNA & 0.89 & $0.46-0.97$ & 1 \\
Mediastinoscopy & 0.81 & $0.78-0.97$ & 1 \\
Mediastinoscopy following & 0.79 & & 10 \\
$\quad$ EBUS-TBNA & & & \\
\hline EBUS-TBNA, Endobronchial ultrasound transbronchial needle aspiration.
\end{tabular}


TABLE 5. Treatment-related mortalities

\begin{tabular}{lcc}
\hline \multicolumn{1}{c}{ Procedure } & Mortality & Reference \\
\hline Chemotherapy/radiation & 0.016 & 15 \\
Mediastinoscopy & 0.0005 & $41-43$ \\
Video-assisted thoracoscopic surgery & 0.01 & $44-46$ \\
\hline
\end{tabular}

We reviewed per-visit fixed and variable costs pertaining to mediastinoscopy and EBUS-TBNA MLNS and VATS lobectomy. We extracted the mean costs for each procedure (Table 3). There were no complications associated with EBUS-TBNA. The costs of managing surgical complications are included in the average procedure cost. Mediastinoscopy average cost was calculated using only cost data from procedures where mediastinoscopy was the only procedure performed.

Costs of medical therapy and related complications, including death, were extracted from the cancer risk management model (CRMM), a web-enabled platform that allows researchers to simulate the influence of different oncologic health policies, such as treatment modalities for common malignancies. ${ }^{15}$ Details regarding the development of the CRMM have been provided elsewhere. ${ }^{11}$ The model was previously validated internally using Statistics Canada data and externally with Canadian Cancer Registry. $^{16}$

For the neoadjuvant therapy regimen we chose the Southwest Oncology Group regimen ${ }^{17}$ currently used at the Princess Margaret Cancer Center, the largest Canadian oncology center. ${ }^{18}$ For the adjuvant chemotherapy, 4 cycles of cisplatinum and vinorelbine were administered 6 to 8 weeks following surgery (Table 3$).^{19}$

Other model parameters. Sensitivity of the EBUS-TBNA and mediastinoscopy for detection of mediastinal lymph node metastasis (MLNM) was based on a recent large systematic review. ${ }^{1}$ Sensitivity of mediastinoscopy following EBUS-TBNA for detection of MLNM was obtained from our institutional data ${ }^{10}$ (Table 4). Procedure or treatmentrelated mortalities were derived from the CRMM (for the chemotherapy and radiation) and from published literature (mediastinoscopy and VATS resections) (Table 5).

\section{Statistical Analysis}

We performed a decision-analytic deterministic cohort simulation. All analyses were performed using the decision-analytic software TreeAge Pro 2015 (TreeAge Software Inc, Williamstown, Mass).

Base-case analysis. To determine comparative clinical effectiveness, we estimated QALYs for each treatment strategy. For the costeffectiveness, we compared clinical outcomes (QALYs) to costs and calculated ICER, comparing the different strategies. ${ }^{20}$

Sensitivity analysis. We performed deterministic 1-way and 2-way sensitivity analyses on influential model parameters. We performed a 1-way sensitivity analysis on prevalence of MLNM varying the prevalence between $0 \%$ and $100 \%$ to determine the optimal staging strategy for different regions of prevalence and a 1-way sensitivity analysis on the sensitivity of EBUS-TBNA varying the sensitivity between $10 \%$ and $89 \%$, based on published evidence. ${ }^{1}$ We performed a 2 -way sensitivity analysis varying simultaneously the prevalence of MLNM and sensitivity of EBUS-TBNA. In our sensitivity analyses comparing results to thresholds of ICERs, we used CAD $\$ 80,000$ per QALY gained as willingnessto-pay threshold (WTP). This value has been endorsed as an oncologic ceiling threshold by the Canadian Expert Drug Advisory Committee and the Cancer Care Ontario Policy Advisory Committee. ${ }^{21,22}$

The study was approved by the University Health Network Research Ethics Board.

\section{RESULTS \\ Base-Case Analysis}

In the comparative effectiveness analysis, the remaining quality-adjusted life expectancies ranged from 5.80 to 5.88 QALYs. Under the base-case scenario, the invasive staging using EBUS-TBNA followed by mediastinoscopy offered the highest QALYs of 5.88, followed by EBUSTBNA staging (5.87 QALYs), mediastinoscopy (5.86 QALYs), and no invasive staging with 5.80 QALYs (Table 6).

In the cost comparison, the least expensive strategy was the no invasive staging, with a cost of CAD $\$ 11,863$, followed by EBUS-TBNA with a cost of $\$ 13,727$, mediastinoscopy with a cost of CAD $\$ 18,143$, EBUS-TBNA with confirmatory mediastinoscopy with a cost of CAD $\$ 19,587$, EBUS-TBNA in operating room with a cost of CAD \$20,551 and EBUS-TBNA in the operating room with confirmatory mediastinoscopy with a cost of CAD $\$ 26,440$ (Table 6).

The cost-effectiveness plane in Figure 1 shows the results of considering both costs and health outcomes associated with each strategy. The strategy in the lower left corner is less expensive and less effective than the strategy in the right upper corner. After eliminating the dominated strategies (a strategy is considered to be dominated if it is more costly and less effective than other nondominated strategies) (mediastinoscopy, EBUS-TBNA in the operating room, and EBUS-TBNA in the operating room with mediastinoscopy) 3 strategies remained, defining the costeffectiveness frontier: no invasive strategy, EBUS-TBNA,

TABLE 6. Cost-effectiveness results, base-case analysis

\begin{tabular}{|c|c|c|c|c|c|}
\hline Strategy & Cost* $^{*}$ & $\begin{array}{c}\text { Incremental } \\
\text { cost }^{*}, \dagger\end{array}$ & $\begin{array}{c}\text { Effectiveness } \\
\text { (QALY) }\end{array}$ & $\begin{array}{c}\text { Incremental } \\
\text { effectiveness (QALY) }\end{array}$ & ICER \\
\hline No invasive staging strategy & 11,863 & 0 & 5.802 & 0 & 0 \\
\hline EBUS-TBNA & 13,727 & 1864 & 5.873 & 0.071 & 26,254 \\
\hline Mediastinoscopy & 18,143 & 4415 & 5.864 & Dominated & Dominated \\
\hline EBUS-TBNA followed by mediastinoscopy & 19,587 & 5860 & 5.877 & 0.004 & $1,426,019$ \\
\hline EBUS-TBNA in the OR & 20,551 & 963 & 5.873 & Dominated & Dominated \\
\hline EBUS-TBNA in the OR followed by & 26,440 & 6853 & 5.877 & Dominated & Dominated \\
\hline
\end{tabular}

mediastinoscopy

QUALY, Quality-adjusted life years; ICER, incremental cost-effectiveness ratio (in Canadian dollars/QALY); OR, operating room; EBUS-TBNA, endobronchial ultrasound transbronchial needle aspiration. *Costs reported in Canadian dollars. †Increments are calculated in comparison to the next best nondominated strategy. 


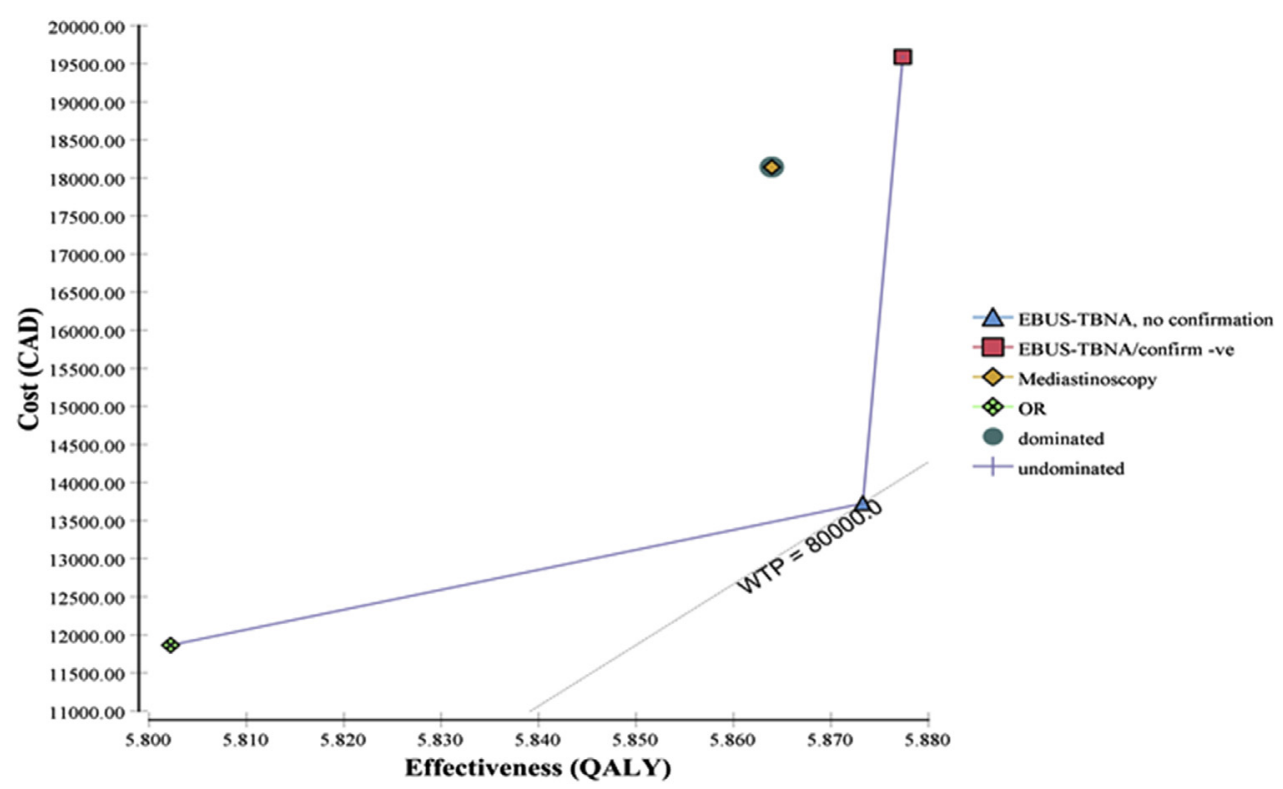

FIGURE 1. Cost-effectiveness plane, base-case analysis. Effectiveness (represented as quality-adjusted life years [QALYS]) is expressed on the $x$ axis, whereas the cost in Canadian dollars $(C A D)$ is expressed on the $y$ axis. The strategy in the lower left corner (no invasive mediastinal staging) is less expensive but also less effective compared with the strategy in the top right corner. After eliminating the dominated strategy, mediastinoscopy (the less effective and more expensive than the preceding strategy), 3 strategies remained defining the cost-effectiveness frontier: no-invasive mediastinal staging $(O R)$, endobronchial ultrasound transbronchial needle aspiration (EBUS-TBNA), and EBUS-TBNA-mediastinoscopy. WTP, Willingness to pay threshold.

and EBUS-TBNA- mediastinoscopy. The ICER of EBUSTBNA compared with no invasive strategy was $\sim 26,000 /$ QALY and the ICER of adding mediastinoscopy to EBUS-TBNA was $\sim 1,400,000 /$ QALY (Table 6).

\section{Sensitivity Analysis}

The noninvasive strategy is a cost-effective strategy up to a MLNM prevalence of $2.5 \%$. Between MLNM prevalence of $2.5 \%$ and $57 \%$, EBUS-TBNA is the preferred and costeffective strategy. Once the MLNM prevalence exceeds $57 \%$, EBUS-TBNA-mediastinoscopy is the preferred, cost-effective strategy.

The 1-way sensitivity analysis on EBUS-TBNA sensitivity shows that mediastinoscopy is not dominated by EBUS-TBNA if the sensitivity of EBUS-TBNA is below $79 \%$. However, it has a high ICER; that is, falling below $\$ 100,000 /$ QALY only if EBUS-TBNA sensitivity is below $19 \%$ and falling below \$80,000/QALY if EBUS-TBNA sensitivity is below $3 \%$. EBUS-TBNA is the preferred strategy and becomes cost-effective if its sensitivity is above $25 \%$.

The 2-way sensitivity analysis evaluated the effect of sensitivity of EBUS-TBNA and prevalence of MLNM. It shows the preferred staging strategy for each combination of MLNM prevalence and EBUS-TBNA sensitivity. For example, with an MLNM prevalence below $11 \%$ and EBUS-TBNA sensitivity below $20 \%$, the noninvasive strategy is the preferred strategy. Once the MLNM prevalence exceeds $11 \%$, mediastinoscopy becomes the preferred strategy and can be considered cost-effective if the sensitivity of EBUS-TBNA is below $20 \%$. The minimum prevalence of MLNM at which confirmatory mediastinoscopy should be added after a negative EBUS-TBNA is around $25 \%$ (if sensitivity of EBUS-TBNA is around $60 \%$ ) (Figure 2).

Analysis with thoracotomy as the preferred surgical approach did not alter conclusions of the analysis. Analysis with EBUS-TBNA performed in the operating room (the option of performing the EBUS-TBNA in the endoscopy was removed from the analysis) altered the analysis significantly. Under the base-case scenario, no invasive staging was cost-effective and this was the preferred strategy. All approaches (mediastinoscopy, EBUS-TBNA, and EBUSTBNA followed by mediastinoscopy) had ICERs above the WTP threshold of $\$ 80,000$ (Table 7 ). In the 2-way sensitivity analysis, evaluating the effect of varying EBUS-TBNA sensitivity and MLNM prevalence, the preferred strategy was the surgical resection without invasive staging, regardless of EBUS-TBNA sensitivity until MLNM prevalence of $\sim 11 \%$. Once the MLNM prevalence exceeded $11 \%$, mediastinoscopy was the preferred staging strategy. EBUS-TBNA with confirmatory mediastinoscopy became cost-effective once the prevalence of MLNM became high $(\sim 70 \%)$ and EBUS-TBNA sensitivity was at least $89 \%$. EBUS-TBNA without confirmatory mediastinoscopy was not cost-effective.

\section{DISCUSSION}

We developed a decision-analytic model to investigate the optimal MLNS pathway for patients with NSCLC 


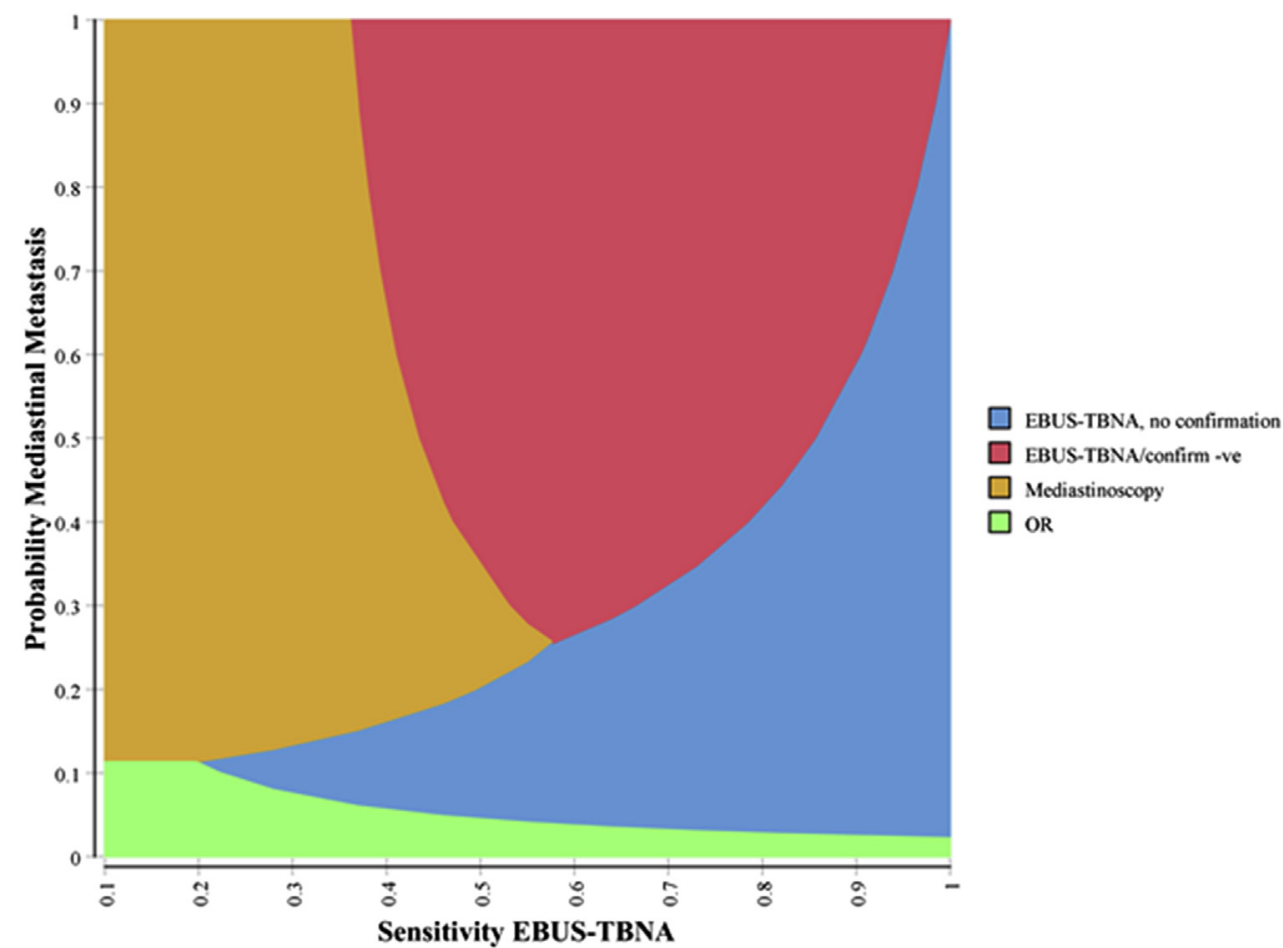

FIGURE 2. Two-way sensitivity analysis on prevalence of mediastinal lymph node metastasis (MLNM) and sensitivity of endobronchial ultrasound transbronchial needle aspiration (EBUS-TBNA) affecting incremental cost-effectiveness of different mediastinal lymph node staging strategies. A willingness-topay threshold of $\$ 80,000$ (Canadian dollars) per quality-adjusted life years gained was used. Colored areas represent regions of different EBUS-TBNA sensitivity and probability of MLNM over which different staging approaches are cost-effective. A staging strategy involving only noninvasive mediastinal lymph node staging, followed by surgical resection $(O R)$ is the preferred staging strategy up until MLNM prevalence of $\sim 11 \%$ if the EBUS-TBNA sensitivity is below 20\%. Once prevalence of MLNM exceeds $11 \%$, and if EBUS-TBNA sensitivity is still below $20 \%$, mediastinoscopy is the preferred staging strategy. EBUS-TBNA staging can be considered test of first choice if its sensitivity is $>20 \%$ and prevalence of MLNM is between $\sim 11 \%$ and $\sim 25 \%$. Once prevalence of MLNM reaches $\sim 25 \%$, confirmatory mediastinoscopy should be considered in patients staged with ENUS-TBNA.

incorporating the most recent, high-quality evidence on performance characteristics of the currently recommended invasive staging strategies, local cost data from a large Canadian tertiary cancer center, and factoring in emerging evidence on the outcomes in patients with micrometastatic mediastinal disease.

In terms of comparative effectiveness, EBUS-TBNA followed by mediastinoscopy is the strategy that results in the highest QALYs. However, it is also the most expensive staging approach. Despite a small survival advantage that the combined procedures provide in the analysis, it results in a prohibitive ICER of $>\$ 1.4$ million/QALY. ${ }^{22}$ Therefore, under our base-case scenario, it may not be justifiable to pursue mediastinoscopy after negative EBUS-TBNA. Staging with EBUS-TBNA alone can be considered costeffective in patients with clinical NO disease and prevalence of MLNM of $9 \%$.

Our 1-way sensitivity analysis provided a more detailed guide to thresholds for implementation of different staging strategies depending on the prevalence of MLNM and sensitivity of EBUS-TBNA, suggesting that invasive MLNS with EBUS-TBNA is not cost-effective if the

TABLE 7. Cost-effectiveness results, base-case analysis with endobronchial ultrasound transbronchial needle aspiration (EBUS-TBNA) performed exclusively in the operating room $(\mathrm{OR})$

\begin{tabular}{|c|c|c|c|c|c|}
\hline Strategy & Cost* & $\begin{array}{c}\text { Incremental } \\
\operatorname{cost}^{*}, \dagger\end{array}$ & $\begin{array}{c}\text { Effectiveness } \\
\text { (QALY) }\end{array}$ & $\begin{array}{c}\text { Incremental } \\
\text { effectiveness (QALY) }\end{array}$ & ICER \\
\hline No invasive staging strategy & 11,863 & 0 & 5.802 & 0 & 0 \\
\hline Mediastinoscopy & 18,143 & 6280 & 5.864 & 0.062 & 101,796 \\
\hline EBUS-TBNA in the OR & 20,551 & 2408 & 5.873 & 0.009 & 258,445 \\
\hline EBUS-TBNA in the OR followed by mediastinoscopy & 26,440 & 5890 & 5.877 & 0.004 & $1,433,233$ \\
\hline
\end{tabular}

QUALY, Quality-adjusted life years; ICER, incremental cost-effectiveness ratio (in Canadian dollars/QALY). *Costs reported in Canadian dollars. †Increments are calculated in comparison to the next best nondominated strategy. 
prevalence of MLNM is $2.5 \%$, but still justifying invasive MLNS in a very-low-risk population (MLNM above $2.5 \%$ ).

This finding is in conflict with current guidelines, which do not advocate invasive MLNS in at least a proportion of patients included in our study (ie, those with clinical stage I disease). ${ }^{1,23}$ The guidelines have been created without factoring in the WTP thresholds and economic data, which may influence the staging recommendation. The aim of our study was to offer clinicians a practical guide to the optimal staging strategy taking under consideration each patient's and the individual program's characteristics. Decision about pursuing invasive MLNS in patients with cN0 disease and a low pretest probability of MLNM $(2.5 \%-10 \%)$ should be made on a case-by-case basis. ${ }^{24}$

We explored the influence institutional variability in EBUS-TBNA sensitivity may have on recommendations for the optimal staging strategy. Our sensitivity analysis suggests that in programs that cannot ensure EBUS-TBNA sensitivity of at least $20 \%$, mediastinoscopy should be the test of first choice for invasive MLNS. This threshold is similar to that reported in prior studies. ${ }^{25}$ Although such a low sensitivity has not been reported in the EBUS-TBNA staging studies, ${ }^{1}$ these studies often come from high volume, tertiary centers where EBUS-TBNA is performed by experts. Our study suggests that ongoing performance quality monitoring is important to ensure the optimal invasive staging.

Another important aspect of MLNS is whether mediastinoscopy should follow negative EBUS-TBNA. Studies show that EBUS-TBNA performance characteristics are similar to mediastinoscopy in MLNS in NSCLC. ${ }^{10,26}$ Guidelines advise confirmatory mediastinoscopy in cases of negative EBUS-TBNA in patients with high pretest probability of MLNM. ${ }^{1}$ In our sensitivity analysis, we addressed the question: At what MLNM prevalence and EBUS-TBNA sensitivity should the confirmatory mediastinoscopy be performed in cases of negative EBUS-TBNA? Our 2-way sensitivity analysis demonstrated that once the prevalence of MLNM reaches approximately $25 \%$ and the EBUSTBNA sensitivity is around $60 \%$, negative EBUS-TBNA should be followed by mediastinoscopy. In cases of lower sensitivity of EBUS-TBNA (and MLNM prevalence at least $25 \%$ ), mediastinoscopy is the test of choice. With even higher prevalence of MLNM, the window for EBUSTBNA-mediastinoscopy opens. For example, in patients with a $50 \%$ prevalence of MLNM, EBUS-TBNAmediastinoscopy is the preferred strategy if the sensitivity of EBUS-TBNA is between $44 \%$ and $88 \%$. Previous studies have shown that extensive preoperative invasive MLNS results in detection of more cases of mediastinal metastasis. ${ }^{10,27}$

It must be emphasized that the true value of confirmatory mediastinoscopy can only be determined by the prevalence of MLNM in the population, the test sensitivities, and the WTP threshold of the country under study.
Meyers and colleagues ${ }^{9}$ looked at mediastinal LN staging in patients with clinical stage I NSCLC, concluding that invasive MLNS is not economically justifiable. However, the study focused on mediastinoscopy as the staging strategy and was performed before the EBUS-TBNA era. Four years following the study by Meyers and colleagues, ${ }^{9}$ Steinfort and colleagues ${ }^{25}$ demonstrated that performing EBUS-TBNA with confirmatory mediastinoscopy if the EBUS-TBNA was negative, was the least costly staging option in a high-risk patient population.

Our study takes the economic analysis further, providing a novel look at the cost-effectiveness of the modalities currently recommended in invasive MLNS. We focused the base-case analysis on a population of patients where there may be some controversy as to whether invasive MLNS should be pursued or not, given the overall low prevalence of MLNM in this population. ${ }^{1,9}$

Our model points out that there is a well-defined role for the use different modalities, including mediastinoscopy. This stresses the need for ongoing focus on maintenance of competency and skill acquisition in mediastinoscopy and EBUS-TBNA by currently practicing and future thoracic surgeons, respectively.

In some programs, EBUS-TBNA is performed in the operating room under general anesthesia. Our costeffectiveness analysis shows that it is more cost-effective to perform mediastinoscopy in patients who need invasive MLNS than to stage patients with EBUS-TBNA performed in the operating room under general anesthesia. Higher than mediastinoscopy, cost of EBUS-TBNA performed in the operating room is consistent with prior literature. ${ }^{28}$ However, EBUS-TBNA performed under conscious sedation is effective, safe, and associated with high patient satisfaction. $^{29-31}$ We hope the results of our study encourage clinicians performing EBUS-TBNA under general anesthesia in an operating room to change their practice.

The question of optimal management of patients with $\mathrm{N} 2$ disease is controversial with no firm guidelines advocating a single management stretegy. ${ }^{1}$ However, evidence is accumulating suggesting that a select patient population with N2 disease may benefit from neoadjuvant therapy followed by surgery over definitive chemotherapy and radiation. $2,3,32,33$ Our study incorporated the influence of trimodality therapy for patients with $\mathrm{N} 2$ disease. We assumed that trimodality therapy offers a survival advantage over definitive chemoradiation. Because the outcome data in the studies incorporating trimodality therapy into the treatment algorithm of patients with $\mathrm{N} 2$ disease comes from a population with clinical N2 disease, we believe that our model, if anything, underestimates the survival in the population of patients we studied because there are no published data on outcomes of trimodality therapy in this patient population. 
As with all decision analyses, our study has several limitations. ${ }^{34}$ Cost-effectiveness analysis is not a complete procedure for determining all decisions in health care because it cannot incorporate all the values, criteria, and individual patient characteristics relevant to such decisions. Rather, it should be used as an aid in the complex decision-making process. ${ }^{35}$

Our model does not account for the QALYs and costs of management of recurrent disease and end-of-life care. This simplification may underestimate costs of diagnostic approaches. However, this is unlikely to substantially influence the differences between the strategies because these costs are similar across strategies following the initial treatment.

In our base-case analysis, we used point estimates for procedure sensitivities. Because these values may be surrounded by uncertainty and affected by specific settings and heterogeneity, we varied these parameters in sensitivity analyses, confirming the robustness of our results.

It must be emphasized that our results may not be generalizable to all clinical scenarios. In some institutions separate TBNA needles may be used for each $\mathrm{LN}$ and rapid onsite evaluation may be used. Each of these situations is expected to increase the per-procedure cost. ${ }^{28}$ In contrast, lower costs might be expected if mediastinal staging is performed as a part of a 2-stage procedure with EBUS-TBNA and/or mediastinoscopy followed by a surgery if the MLNS is negative.

We used overall life expectancies for the QALY calculation. However, life expectancies may differ substantially across patients and may be affected by age, comorbidity, and other factors. Therefore, any individual clinical decision should carefully consider all individual patient characteristics and preferences before the staging decision is made.

The analysis using thoracotomy as the preferred surgical procedure instead of VATS did not alter our study conclusions. However, we did not have separate utilities for patients managed with thoracotomy. Thoracotomy may be associated with a slightly lower QALY. However, given lower than EBUS-TBNA costs of the operating room pathway with thoracotomy, this would not alter our conclusions.

Procedure costs have been based on approximately $10 \%$ of all surgical cases costed at our institution. We realize that this represents a limitation to our study because not all procedure-related costs have been captured. However, the reason for that is administrative and not related to the case itself; therefore, it is unlikely that cases captured and not captured were different in terms of per-procedure cost. In addition, we compared the average cost of the VATS resection from our database CAD $\$ 11,215$ (95\% CI, \$9279$\$ 13,150$ ) with that reported in the CRMM, which was approximately CAD $\$ 12,000$. We therefore believe that our costed cases capture accurately Canadian procedure costs.
We did not account for physician fees because they differ across Canada and would not be applicable to other countries. Finally, we did not account for indirect costs related to patient and caregiver loss of productivity resulting from the disease and during the diagnostic and management periods. Although we believe that it is unlikely that adding these cost would make a major difference for our analysis outcome, taking a full societal perspective and including productivity losses could be a subject of further research.

\section{CONCLUSIONS AND FUTURE DIRECTIONS}

Based on our decision analysis, in patients with clinical N0 disease and estimated low prevalence of MLNM, invasive staging with EBUS-TBNA is cost-effective. The benefit conveyed by detection of mediastinal metastatic disease becomes more apparent as the prevalence of MLNM increases, with confirmatory mediastinoscopy becoming cost-effective in cases of negative EBUS-TBNA in patients with moderate to high probability of MLNM. Performing EBUS-TBNA in the operating room is not cost-effective, and from a health economics perspective if there is no ability to perform EBUS-TBNA in the endoscopy, the more cost-effective strategy is to offer mediastinoscopy to patients who need invasive MLNS.

Our model provides an evaluation of the best-case scenario using the currently available best evidence on procedure performance characteristics, life expectancies, utilities, and costs. However, a revised estimate of cost-effectiveness of aggressive preoperative assessment of patients with lung cancer should be generated once more, and hopefully prospectively collected, real-world data from large cohorts of patients with micrometastatic mediastinal disease treated with trimodality become available.

\section{Conflict of Interest Statement}

K.C.-K. is a research consultant with Olympus America, and K.Y. is a research consultant and has received research support educational grants from Olympus America. All other authors have nothing to disclose with regard to commercial support.

\section{Webcast}

You can watch a Webcast of this AATS meeting presentation by going to: http://webcast.aats.org/2016/Video/ Tuesday/05-17-16_Hall_E_0925_Czarnecka-Kujawa-800. mp4.

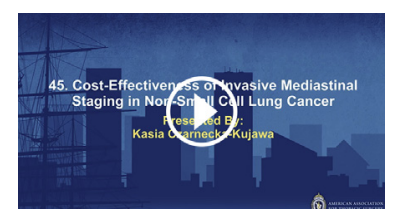


The authors thank the Competence Centers for Excellent Technologies (COMET Center) Center for Personalized Cancer Medicine (ONCOTYROL) for contributing to our work. COMET Center ONCOTYROL is funded by the Austrian Federal Ministries Federal Ministry for Transport, Innovation and Technology (BMVIT)/Federal Ministry of Economy, Family and Youth (BMWFJ) (via Austrian Research Promotion Agency [FFG]) and the Tiroler Zukunftsstiftung/Standortagentur Tirol.

\section{References}

1. Silvestri GA, Gonzalez AV, Jantz MA, Margolis ML, Gould MK, Tanoue LT, et al. Methods for staging non-small cell lung cancer: diagnosis and management of lung cancer, 3rd ed: American College of Chest Physicians evidence-based clinical practice guidelines. Chest. 2013;143(5 Suppl):e211S-50S.

2. Uy KL, Darling G, Xu W, Yi QL, De Perrot M, Pierre AF, et al. Improved results of induction chemoradiation before surgical intervention for selected patients with stage IIIA-N2 non-small cell lung cancer. J Thorac Cardiovasc Surg. 2007;134:188-93

3. Darling GE, Li F, Patsios D, Massey C, Wallis AG, Coate L, et al. Neoadjuvant chemoradiation and surgery improves survival outcomes compared with definitive chemoradiation in the treatment of stage IIIA N2 non-small-cell lung cancer. Eur J Cardiothorac Surg. 2015;48:684-90; discussion 690.

4. Siebert U. When should decision-analytic modeling be used in the economic evaluation of health care? Eur J Health Econ. 2003;4:143-50.

5. Caro JJ, Briggs AH, Siebert U, Kuntz KM. Modeling good research practicesoverview: a report of the ISPOR-SMDM Modeling Good Research Practices Task Force-1. Med Decision Making. 2012;32:667-77.

6. Caro JJ, Briggs AH, Siebert U, Kuntz KM. Modeling good research practicesoverview: a report of the ISPOR-SMDM Modeling Good Research Practices Task Force-1. Value Health. 2012;15:796-803.

7. Eddy DM, Hollingworth W, Caro JJ, Tsevat J, McDonald KM, Wong JB. Model transparency and validation: a report of the ISPOR-SMDM Modeling Good Research Practices Task Force-7. Med Decision Making. 2012;32:733-43.

8. Husereau D, Drummond M, Petrou S, Greenberg D, Mauskopf J, Augustovski F, et al. Reply to Roberts, et al.: CHEERS is sufficient for reporting cost-benefit analysis, but may require further elaboration. Pharmacoeconomics. 2015;33: 535-6.

9. Meyers BF, Haddad F, Siegel BA, Zoole JB, Battafarano RJ, Veeramachaneni N, et al. Cost-effectiveness of routine mediastinoscopy in computed tomographyand positron emission tomography-screened patients with stage I lung cancer. J Thorac Cardiovasc Surg. 2006;131:822-9; discussion 822-9.

10. Yasufuku K, Pierre A, Darling G, de Perrot M, Waddell T, Johnston M, et al. A prospective controlled trial of endobronchial ultrasound-guided transbronchial needle aspiration compared with mediastinoscopy for mediastinal lymph node staging of lung cancer. J Thorac Cardiovasc Surg. 2011;142:1393-400.e1391.

11. Louie AV, Senan S, Dahele M, Slotman BJ, Verbakel WF. Stereotactic ablative radiation therapy for subcentimeter lung tumors: clinical, dosimetric, and image guidance considerations. Int J Radiation Oncol Biol Physics. 2014;90:843-9.

12. McIntosh CN, Connor Gorber S, Bernier J, Berthelot JM. Eliciting Canadian population preferences for health states using the Classification and Measurement System of Functional Health (CLAMES). Chron Dis Can. 2007:28:29-41.

13. Evans WK, Connor Gorber S, Spence ST, et al. Health state descriptions for Canadians: cancers. Canada Statistics Canada; 2005. Available at: http://www. statcan.gc.ca/access_acces/alternative_alternatif.action? $1=$ eng \&loc=82-619m2005001-eng.pdf. Accessed May 1, 2016.

14. Bank of Canada. Inflation calculator. Available at: http://www.bankofcanada.ca/ rates/related/inflation-calculator/. Accessed May 13, 2015.

15. Canadian Partnership Against Cancer Corporation. Cancerview.ca Web site. Available at: http://www.cancerview.ca. Accessed February 1, 2016.

16. Evans WK, Wolfson MC, Flanagan WM, et al. The evaluation of cancer control interventions in lung cancer using the Canadian Cnacer Risk Management Model. Lung Cancer Mgmt. 2012;25-33.

17. Albain KS, Rusch VW, Crowley JJ, Rice TW, Turrisi AT III, Weick JK, et al. Concurrent cisplatin/etoposide plus chest radiotherapy followed by surgery for stages IIIA (N2) and IIIB non-small-cell lung cancer: mature results of Southwest Oncology Group phase II study 8805. J Clin Oncol. 1995;13:1880-92.

18. The Princess Margaret Cancer Centre, University Health Network. Lung site group. Available at: http://www.uhn.ca/PrincessMargaret/Health_Professionals/Programs_ Departments/Lung. Accessed February 8, 2016.
19. The Princess Margaret Cancer Centre, University Health Network. Clinical practice guidelines: lung cancer. Available at: http://www.uhn.ca/PrincessMargaret/Health_ Professionals/Programs_Departments/Lung/Pages/clinical_practice_guidelines.aspx. Accessed February 8, 2016.

20. Weinstein MC, Stason WB. Foundations of cost-effectiveness analysis for health and medical practices. N Engl J Med. 1977;296:716-21.

21. Rocchi A, Menon D, Verma S, Miller E. The role of economic evidence in Canadian oncology reimbursement decision-making: to lambda and beyond. Value Health. 2008;11:771-83.

22. Schwarzer R, Rochau U, Saverno K, Jahn B, Bornschein B, Muehlberger N, et al. Systematic overview of cost-effectiveness thresholds in ten countries across four continents. J Compar Effectiveness Res. 2015;4:485-504.

23. Darling GE, Dickie AJ, Malthaner RA, Kennedy EB, Tey R. Invasive mediastinal staging of non-small-cell lung cancer: a clinical practice guideline. Curr Oncol (Toronto). 2011;18:e304-310.

24. Darling GE, Maziak DE, Clifton JC, Finley RJ. The practice of thoracic surgery in Canada. Can J Surg. 2004;47:438-45.

25. Steinfort DP, Liew D, Conron M, Hutchinson AF, Irving LB. Cost-benefit of minimally invasive staging of non-small cell lung cancer: a decision tree sensitivity analysis. J Thorac Oncol. 2010;5:1564-70.

26. Ernst A, Anantham D, Eberhardt R, Krasnik M, Herth FJ. Diagnosis of mediastinal adenopathy-real-time endobronchial ultrasound guided needle aspiration versus mediastinoscopy. J Thorac Oncol. 2008;3:577-82.

27. Annema JT, van Meerbeeck JP, Rintoul RC, Dooms C, Deschepper E, Dekkers OM, et al. Mediastinoscopy vs endosonography for mediastinal nodal staging of lung cancer: a randomized trial. JAMA. 2010;304:2245-52.

28. Andrade RS, Podgaetz E, Rueth NM, Majumder K, Hall E, Saric C, Thelen L. Endobronchial ultrasonography versus mediastinoscopy: a single-institution cost analysis and waste comparison. Ann Thorac Surg. 2014;98:1003-7.

29. Lee JE, Kim HY, Lim KY, Lee SH, Lee GK, Lee HS, Hwangbo B. Endobronchial ultrasound-guided transbronchial needle aspiration in the diagnosis of lung cancer. Lung Cancer (Amsterdam). 2010;70:51-6.

30. Kang HJ, Hwangbo B, Lee GK, Nam BH, Lee HS, Kim MS, et al. EBUS-centred versus EUS-centred mediastinal staging in lung cancer: a randomised controlled trial. Thorax. 2014;69:261-8.

31. Steinfort DP, Irving LB. Patient satisfaction during endobronchial ultrasoundguided transbronchial needle aspiration performed under conscious sedation. $R e$ spir Care. 2010;55:702-6.

32. Aggarwal C, Li L, Borghaei H, Mehra R, Somaiah N, Turaka A, et al. Multidisciplinary therapy of stage IIIA non-small-cell lung cancer: long-term outcome of chemoradiation with or without surgery. Cancer Control. 2014;21:57-62.

33. Albain KS, Swann RS, Rusch VW, Turrisi AT III, Shepherd FA, Smith C, et al. Radiotherapy plus chemotherapy with or without surgical resection for stage III non-small-cell lung cancer: a phase III randomised controlled trial. Lancet. 2009; 374:379-86

34. Gazelle GS, McMahon PM, Siebert U, Beinfeld MT. Cost-effectiveness analysis in the assessment of diagnostic imaging technologies. Radiology. 2005;235: $361-70$.

35. Russell LB, Gold MR, Siegel JE, Daniels N, Weinstein MC. The role of costeffectiveness analysis in health and medicine. Panel on Cost-Effectiveness in Health and Medicine. JAMA. 1996;276:1172-7.

36. Harpole DH Jr, Herndon JE II, Young WG Jr, Wolfe WG, Sabiston DC Jr. Stage I nonsmall cell lung cancer. A multivariate analysis of treatment methods and patterns of recurrence. Cancer. 1995;76:787-96.

37. Port JL, Kent MS, Korst RJ, Libby D, Pasmantier M, Altorki NK. Tumor size predicts survival within stage IA non-small cell lung cancer. Chest. 2003;124 1828-33.

38. Keller SM, Adak S, Wagner H, Herskovic A, Komaki R, Brooks BJ, et al. A randomized trial of postoperative adjuvant therapy in patients with completely resected stage II or IIIA non-small-cell lung cancer. Eastern Cooperative Oncology Group. N Engl J Med. 2000;343:1217-22.

39. Martini N, Burt ME, Bains MS, McCormack PM, Rusch VW, Ginsberg RJ. Survival after resection of stage II non-small cell lung cancer. Ann Thorac Sug. 1992; 54:460-5; discussion 466.

40. Douillard JY, Rosell R, De Lena M, Carpagnano F, Ramlau R, GonzalesLarriba JL, et al. Adjuvant vinorelbine plus cisplatin versus observation in patients with completely resected stage IB-IIIA non-small-cell lung cancer (Adjuvant Navelbine International Trialist Association [ANITA]): a randomised controlled trial. Lancet Oncol. 2006;7:719-27.

41. Lemaire A, Nikolic I, Petersen T, Haney JC, Toloza EM, Harpole DH Jr, et al. Nine-year single center experience with cervical mediastinoscopy: 
complications and false negative rate. Ann Thorac Surg. 2006;82:1185-9; discussion 1189-90.

42. Hammoud ZT, Anderson RC, Meyers BF, Guthrie TJ, Roper CL, Cooper JD, Patterson GA. The current role of mediastinoscopy in the evaluation of thoracic disease. J Thorac Cardiovasc Surg. 1999;118:894-9.

43. Zakkar M, Tan C, Hunt I. Is video mediastinoscopy a safer and more effective procedure than conventional mediastinoscopy? Interact Cardiovasc Thorac Surg. 2012;14:81-4.

44. Allen MS, Darling GE, Pechet TT, Mitchell JD, Herndon JE II, Landreneau RJ, et al. Morbidity and mortality of major pulmonary resections in patients with early-stage lung cancer: initial results of the randomized, prospective ACOSOG Z0030 trial. Ann Thorac Surg. 2006;81:1013-9; discussion 1019-20.

45. D'Amico TA. Long-term outcomes of thoracoscopic lobectomy. Thorac Surg Clin. 2008;18:259-62.

46. Louie BE, Wilson JL, Kim S, Cerfolio RJ, Park BJ, Farivar AS, Vallieres E, et al. Comparison of video-assisted thoracoscopic surgery and robotic approaches for clinical stage I and stage II non-small cell lung cancer using the Society of Thoracic Surgeons database. Ann Thorac Surg. 2016;102:917-24.

Key Words: cost-effectiveness, mediastinal lymph node staging, EBUS-TBNA, mediastinoscopy

\section{Discussion}

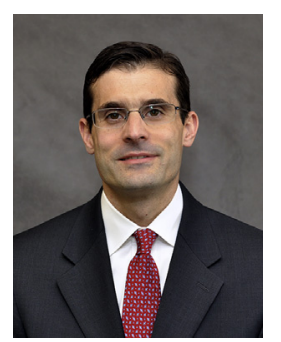

Dr Felix G. Fernandez (Atlanta, Ga). In this decision analysis, examining the cost-effectiveness of invasive mediastinal staging in patients with clinical N0 non-small cell lung cancer, the investigators conclude that endobronchial ultrasound (EBUS) with transbronchial needle aspiration (TBNA) is cost-effective even with a low estimated prevalence of mediastinal lymph node metastases.

The sensitivity of a test is related to the prevalence of disease in the population studied. The Toronto group has previously reported an $81 \%$ sensitivity for EBUS in a cohort of patients with a $35 \%$ prevalence of mediastinal lymph node metastases. However, the prevalence of mediastinal lymph node metastases in clinical stage I lung cancer after staging by positron emission tomography and computed tomography is approximately $3 \%$ to $6 \%$, and the sensitivity of EBUS would be expected to be much lower in this setting. For reference, mediastinoscopy has $25 \%$ to $40 \%$ sensitivity in this scenario.

Based on the 2-way sensitivity analysis presented, if the prevalence of mediastinal lymph node metastases is $5 \%$ and the sensitivity of EBUS drops to $50 \%$, EBUS is not costeffective and the number needed to treat to detect 1 mediastinal lymph node metastasis is 40 .

I have 3 questions. What is the sensitivity of EBUS in patients with a clinical N0 lung cancer staged by positron emission tomography-computed tomography imaging both at your center of excellence and more broadly across North America?

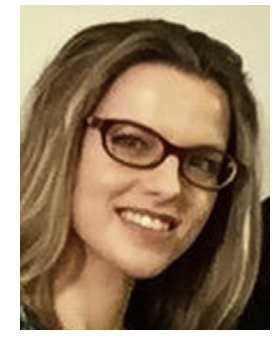

Dr Czarnecka-Kujawa. Thank you. Great question. The majority of published data on performance of EBUSTBNA in lung cancer staging come from large centers of excellence. We have now 2 large meta-analyses looking at this.

We do not really have information on EBUS performance in smaller and community centers that are just starting to use this technology for mediastinal staging. In addition, despite large amounts of data that have accumulated on the topic, there is paucity of data on EBUS-TBNA performance in the population with clinical stage I (T1 N0) disease, simply because invasive staging is not commonly done in this patient population.

The data that are available show that some centers of excellence are able to achieve a very high yield for the procedure with sensitivity nearing $90 \%$, and the performance at our center is consistent with these data.

While creating the model, what we wanted to do is to create the best-case scenario. However, a limitation of this approach is that we are not able to account for all of the variations of clinical outcomes that could be encountered. Therefore, our findings should be interpreted with caution by centers with different patient characteristics and different test performance characteristics from the ones included in our model.

I think a very powerful feature of decision analysis is the fact that one can simulate a variety of different clinical scenarios in sensitivity analysis. That is what we accomplished with the 1-way sensitivity analysis on EBUS-TBNA sensitivity and the 2-way sensitivity analysis on EBUS-TBNA sensitivity and prevalence of mediastinal nodal metastasis to allow clinicians from centers all over the world, with test performance characteristics different from our center's unique program and patient characteristics, to use our work as a guide to offer the best possible mediastinal lymph node staging strategy to their patients.

Dr Fernandez. Thank you. Based on your data, do you recommend that all patients with a clinical stage T1 N0 non-small cell lung cancer undergo mediastinal staging with EBUS, or do you think this should be reserved for patients with larger or more central tumors?

Dr Czarnecka-Kujawa. Thank you for another great question. No, I would not advocate invasive mediastinal lymph node staging in all patients with clinical stage I disease (T1 N0).

Our model shows that it is cost-effective to stage patients with clinical stage I disease. However, cost-effectiveness should not be equated with an automatic recommendation to invasively stage everybody. If anything, we would like to provide guidance to physicians, to use the best staging 
strategy based on their patient population and test performance characteristics.

We follow clinical guidelines at our center. Consistent with that, currently, we do not perform routine invasive staging in patients with clinical stage I (T1 N0) disease. Staging of these patients occurs on occasion, and only as a part of a clinical trial. In my opinion, each center should assess the need for invasive staging in these patients on a case-by-case basis.

Dr Fernandez. Great. Your study assumes a 50\% survival benefit for neoadjuvant therapy versus adjuvant therapy in the setting of occult mediastinal lymph node metastases. A benefit for induction therapy in patients with occult $\mathrm{N} 2$ disease has not been proven.

Have you considered a sensitivity analysis to determine how the magnitude of potential benefit of induction therapy influences the cost-effectiveness of invasive mediastinal staging?

Dr Czarnecka-Kujawa. That is another great question. We have not performed sensitivity analysis and survival benefit, but it is definitely something that we are interested in doing in the future.

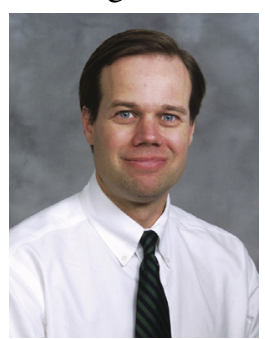

Dr David H. Harpole (Durham, NC). This was a wonderful study, and I think it supports the guidelines for patients with early stage cancer that if the prevalence is under $10 \%$, probably direct a surgery, and in a noninvasive stage it is probably not indicated.

My question to you, and I think you alluded to this, is that in centers of excellence, the type of EBUS-fine needle aspiration done may not be equivalent to that done in the community, and that multistation, multinodal EBUS is what you are talking about where one is actually assessing the entire mediastinum as opposed to, unfortunately, sometimes what we see in a community is that they go after a single node, which is not equivalent to your type of EBUS. I wondered if you could comment on that.

Dr Czarnecka-Kujawa. To call EBUS-TBNA procedure staging, you have to try and recreate what the previous gold standard-cervical mediastinoscopy-was accomplishing. So I think sampling of 1 lymph node is only justifiable for bulky clinical N3 or N2 disease or in a patients with infiltration of the mediastinum by the tumor where the goal is to confirm the diagnosis and get enough tissue for molecular testing.

To label EBUS-TBNA mediastinal lymph node sampling as staging, in other than the population mentioned above, you really have to go systematically, and assess all lymph nodes that mediastinoscopy would, including N3 lymph nodes that will not be sampled during surgery.

In my opinion, if you know that your test performance characteristics are not consistent with what you would be able to accomplish with mediastinoscopy, then either referring to another center for EBUS-TBNA or proceeding with mediastinoscopy at your center is what patients should get to meet the definition of guideline-concordant care. This is also the most cost-effective approach based on our model's prediction.

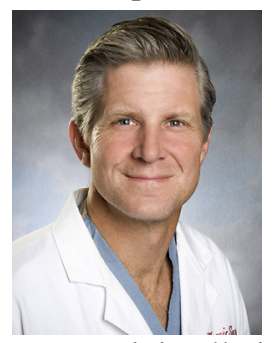

Dr Scott J. Swanson (Boston, Mass). I enjoyed your talk quite a bit. In following-up Dr Fernandez's comment, if the prevalence is somewhere around 5\%, would you ever consider an option where you do mediastinal ipsilateral staging at the beginning of the operation to resect the tumor minimally invasively? And in the 1 in 20 or so cases that are positive, you could stop your video-assisted thoracoscopic surgery with 2 or 3 small incisions and give neoadjuvant therapy, and that way you'd save an extra procedure and a lot of extra time. Would that ever be something you would consider in your algorithm?

Dr Czarnecka-Kujawa. At our center, all lung cancer resections (except for wedge resections), including those for clinical stage I disease are routinely accompanied by lymphadenectomy. However, preoperative mediastinal staging with mediastinoscopy is not routinely done in this patient population (clinical T1 N0).

However, if EBUS is negative in a high-risk patient; for example, in a patient who has a clinical N1, N2, or N3 disease (positive positron emission tomography-computed tomography or computed tomography preoperative staging), we would book these patients for a combined procedure where mediastinoscopy would be performed before lung resection as a part of a 2- stage procedure, where lung resection would follow mediastinoscopy if quick sections of mediastinal lymph nodes are negative. If the staging is positive on mediastinoscopy, then the lung resection does not take place. To summarize, we do not routinely invasively stage clinical stage I patients with negative EBUS, negative positron emission tomography-computed tomography, and computed tomography chest images.

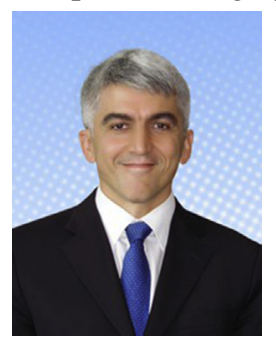

Dr Akif Turna (Istanbul, Turkey). Thank you for the nice presentation. During the creating of your model, what were the considerations regarding the type of mediastinoscopy, namely videoassisted mediastinoscopy, video-assisted mediastinal lymphadenectomy, or standard mediastinoscopy?

What were the considerations regarding the used needles during EBUS and the length of stay after mediastinoscopy? Did you look at the results in terms of different histologic types; for example, adenocarcinoma and squamous cell carcinoma? Because it is fair to propose that in adenocarcinoma the sensitivity and the false negativity would be different than with squamous cell carcinoma. 
Dr Czarnecka-Kujawa. Sorry, the first question was?

Dr Turna. The number of needles used, the type of mediastinoscopy, and the length of stay after mediastinoscopy.

Dr Czarnecka-Kujawa. Sure. So first you were asking for type of mediastinoscopy. The data we used were for cervical mediastinoscopy.

Dr Turna. Was it standard?

Dr Czarnecka-Kujawa. No, video-assisted.

Dr Turna. Video, okay. In terms of length of stay, at our center mediastinoscopy is done as a day procedure. Patients are discharged home the same day. Additional costs associated with rare cases of postmediastinoscopy complications and resulting hospital admission are factored into our model.

Dr Czarnecka-Kujawa. Regarding the number of needles used for our EBUS-TBNA procedures, we try to minimize the number of needles used. For that reason, we start with N3 lymph nodes and proceed systematically to lymph nodes with increased risk of being involved with metastatic disease (N2 and N1 in some cases). If there is a high suspicion of a lymph node being positive and we do not have the rapid onsite evaluation, we change needles. But generally we are trying to accomplish the procedure with the use of as few needles as possible. The use of extra needles, and the associated additional cost, have been factored in to our model.

Dr Turna. Histologic type?

Dr Czarnecka-Kujawa. And I think you were asking also about the sensitivities of the procedure based on tumor histology. We have not been able to separate the procedure yield based on tumor histology.

Dr Moon. This has been an excellent presentation. Beautifully done. But to get the final clinical influence, I'm going to ask how this is going to change things at Emory.

Dr Fernandez. In patients with a clinical N0 lung cancer, the number needed to detect 1 occult N2 node with EBUS is going to be in the range of 25 to 40 patients or higher, and that coupled with the unproven benefit of induction therapy in the setting of an occult mediastinal lymph node metastases, keeps us from applying this strategy routinely in patients at our center. Thank you. 